\title{
Contribution à l'étude de la couche limite des houles monochromatiques
}

\section{$\wedge$ contribution to the study of the boundary layer of monochromatic water waves}

\author{
PAR P. LHERMTTTEE \\ INGINTEUn DES PONTS FP CHAUSSIES
}

\begin{abstract}
Liobservation par colorimétrie des houles de laboratoire, cylindriques monochromatiques, permet de mattro en évidence l'existence et les caracteristiques d'une zone située près du fond directement influence par le contact de la paroi et qui constitue la « couche limite » de ces mouvements.

L'étude experimentale et théorique de la couche limite des houles monochromaliques permet de rendre compte de certains phénomines marginanx et de préciser les conditions aux limiles determinant le monvement du fluide sain. En particulier, le développement et la stabilité des phénomènes de conche limile ainsi que l'évolution de la turbulence dans le fluide sain it partir de la conche limite semblent pounoir etre déterminés en fonction de parametres sans dimensions qui devraient être considérés comme caracteristique imporfante du mouvement.
\end{abstract}

\begin{abstract}
The colorimetric observation of cylindrical, monochromalic laboratory waler waves demonstrates the existence and characteristics af a region near the bottom which is directly infuenced bly contact wilh the wall and which forms the "boundary layer" for this motion. Experimental and theoretical investigations of the boundary layer of monochromatic water manes, bring ont certain marginal phenomena and define the boundary conditions which determine the motion of the unaffected fluid.

In particular, it seems that the development and stability of boundary latjer phenoment as well as the evolution of turbulence in the unaffected fluid can be delermined in terms of dimensionless perameters which should be considered as an important characteristic of the motion.
\end{abstract}

\section{INTRODUCTION}

Les connaissances actuelles en ce qui concerne la houle eylindrique de laboratoire permettent de déterminer la forme mathémalique des solutions les plus générales salisfaisant à l'équation de l'hydrodynamique classique relative a un fluide parfait non doué de viseosité el animé d'un mouvement non turbulent. Les vérifications expérimentales sont, dans ce domaine, en excellent accord avec la théorie, et l'on peut admettre que les différentes solutions permettent, avec l'approximation que l'on désire, de décrire de facon très satisfaisante les mouvements du fluide dans son ensemble.
Toutefois, la solution donnée par les équations de l'hydrolynamique classique exige d'admette que la composante horizonlate des parlirales de la vilesse a la paroi esl finie. Celle hypothese est en fait incompatible avee les propriétés des fluides réels el il semble nécessaire d'étudier de plus près les phénomines marginaux, c'est-à-dire le développement du mouvement dans la zone situce près de la paroi. Les proprićtés caractéristiques du mouvement dans cette zone marginale proviennent du fait que la nullité de la vitesse à la paroi, exigsée par les conditions aux limites, entraine l'existence d'un 
adient élevé des vitesses dans une zone relatisment limitée et, en conséquence, ne permet us de négliger les effets de la viscosité. Il s'agit, en fait, de la « couche limite» lié an développement du mourement du fluide.

\section{I. - DESCRIPTION DES PHÉNOMENES DE COUCHE LIMITE}

\section{CONDITIONS D'EXÉcutron DES ESSAIS :}

Les essais ont été effectués au Laboratoire entral d'Hydraulique de France dans différents maux à houle de ce laboratoire. Les expérienss ont porté sur des houles cylindriques aussi ures que possible, dont les longueurs d'onde it varié de 0,5 à $8 \mathrm{~m}$, les hauteur's d'eau de 10 $50 \mathrm{~cm}$ et les amplitudes de 1 à $26 \mathrm{~cm}$.

Cing types de rugosités différentes ont été udiés, s'échelonnant entre une rugosité nulle laque de verre) et une rugosité moyenne de $\mathrm{mm}$.

La couche limite engendrée dans ces divers ouvements, et dans les différents cas expérientaux envisagés, a été étudiée, soit sur fond srizontal, soit en présence de pente variant de à $5 \%$.

La méthode expérimentale a essentiellement msisté en une observation visuelle par méthode lorimétrique, des phénomènes situés près de paroi ainsi que de l'influence des phénomènes ? couche limite sur le mouvement du fluide in.

\section{COUCHE limite LAMINAIRE:}

Tant que l'amplitude du mouvement ne déisse pas une certaine valeur critique, on peut server des couches limites près du fond paritement laminaires. Il est aisé de mettre en idence, dans ce domaine, la conche limite lainaire, par la méthode colorimétrique, car lle-ci est très nettement différentiable du fluide luée hors de son domaine par sa coloration tense et surtout par la vilesse moyenne d'enainement qu'elle présente.

En effet, la particularité essentielle des coules limites laminaires consiste dans l'existence une vitesse d'entrainement moyen dans le sens : propagation de la houle correspondant à un bit de fluide relativement important dans la ne située près du fond. Cette particularité, qui rmet de mettre en évidence la couche limile, cours des expériences, ne peut s'expliquer jar ; théories classiques de la houle. Elle semble :e liée étroitement à la manifestation des fors de viscosité dans la couche limite.

Lorsque l'on observe attentivement la couche nite d'une houle cylindrique de laboratoire, observe en plus de ce débit moyen une "res- piration » de la couche limite correspondant à une variation d'épaisseur au cours du temps. Enfin, d'une façon très génçale, au cours du mouvement retour des particules du fluide, on observe également un décollement caractéristique de la couche limite.

Comme nous le verrons par la suite, les considérations théoriques concernant la couche limite laminaire des mouvements permettent de rendre comple de facon satisfaisante de ces diver's phéomènes.

\section{c) Covche limite turbulente :}

Lorsque l'amplitude de la houle est suffisamment élevée et lorsque la rugosité du fond est suffisamment forte, la couche limite est inslable et l'observation colorimétrique met en évidence une émission particulièrement active de turbulence près du fond du canal. Il s'agit alors d'une couche limite turbulente dont les mouvements moyens d'entrainement sont beaucoup moins importants que ceux observés au cours de l'étude des couches limites laminaires et fortement aléatoires, pouvant d'ailleurs être dirigés, soit dans le sens de propagation de la houle, soit dans le sens contraire.

L'émission de turbulence provenant de l'instabilité du mouvement, par suite de l'action de la viscosité dans la zone oú se développe la couche limite, se traduit par une contamination du lluide sain qui devient progressivement également tarbulente sur une hautenr importante.

Nous avons ćtudie, au cours de nos expériences, ces divers phénomenes et donné des valeurs pratiques de leur domaine d'existence.

\section{d) CovGh: haMT: PARTEL LEMENT TURBULENT: :}

Avant dobserver une couche limile totalement turbulente, on peul observer des couches limites présentant des phases sporadiques d'instabilite (émission de bouffées turbulentes), tandis que le mouvement dans son ensemble comporte les caractéristiques d'une couche limite laminaire (mouvement moyen dans le sens de la propagation de la houle, décollement, hauteur variable au cours d'une période de mouvement). Il semble que ce phénomène ne constitue pas uniquement un phénomène de transition entre la cou- 
che limite laminaire et la couche limite turbulente, mais qu'il dépend, non seulement des conditions de stabilité près du fond, mais également de l'influence du mouvement général du fluide sur la stabilité de la couche limite.

Nous abordons ici un caractère particulier des couches limites des mouvements à déplacement moyen négligeable, pour lesquelles l'influence du mouvement du fluide sain semble aussi importante sur la couche limite que l'influence du fond. Dans le cas particulier des houles, de nombreux phénomènes exigent, pour pouvoir être compris, de ne pas perdre de vue que le mouvement orbitaire caractéristique de la houle, qui correspond à un mouvement particulièrement stable par suite de sa faible consommation d'énergie du fluide sain, comporte une certaine « rigidité » qui impose son influence sur les mouvements de la couche limite d'une facon comparable à l'influence de la paroi.

\section{e) INFluence de LA COUCHE LimUte SUR LE MOU- VEMENT DU FLUIDE SAIN :}

Si le fluide sain exerce une influence importante sur le développement de la couche limite, réciproquement, le mouvement de celui-ci ne peut pas être étudié séparément de celui de la couche limite. Nous avons déjà dit que dans le cas d'une couche limite totalement turbulente, l'existence de celle-ci entrainait une émission continue de turbulence dans la zone du mouvement du fluide sain, e'est-ì-dire dans la zone du mouvement où la viscosité du fluide ne constitue pas un facteur d'émission de turbulence, mais, ainsi que nous le verrons plus loin, constitue, au contraire, un facteur d'évolution de la turbulence. Dans le cas de couche limite laminaire, l'existence de couche limite correspondant à la zone où se concentrent les actions de frottement contre la paroi, entraîne l'existence d'un rotationnel décroissant depuis le fond jusqu'en surface dans le mouvement du fluide sain. On sait, depuis les travaux de M. Miche, de Mme Dubreuil-Jacotin et de M. Biesel, que l'existence du rotationnel correspond à une certaine distribution du courant moyen (courant du second ordre par rapport à l'amplitude).

M. Miche [8] a montré que la distribution du rotationnel dans les houles normales pouvait ètre déterminée à partir de la connaissance du frottement de l'air sur la surface libre du mouvement. De mème, la connaissance de la propagation de la couche limite permet de déterminer le rotationnel lié au frottement sur la paroi. La distribution du rotationnel dans une houle n'est done pas arbitraire, mais est déterminée par les conditions aux limites.

\section{II. - ÉTUDE THÉORIQUE DE LA COUCHE LIMITE LAMINAIRE}

\section{a) Définitron mathématique:}

La définition mathématique de la couche limite des houles, telle qu'elle a été suggérée par M. Huon Li dans son étude [4], ne permet pas de rendre compte des propriétés de la couche limite des houles telle que nous l'avons observée.

Il semble qu'il soit possible, à partir des théories mathématiques, de donner une définition théorique de la couche limite dans le cas des mouvements périodiques, qui s'accorde mieux avec l'expérience que la définition ci-dessus.

En effet, la recherche d'une expression de la vitesse dans la couche limite satisfaisant à l'équation de Navier-Stokes dans le cas de mouvements périodiques, permet de définir une épaisseur o $(b t-a x)$ pour laquelle la vitesse à la frontière de la couche limite est identiquement égale à la vitesse dans le fluide sain.

Les calculs conduisent alors à définir une couche limite d'épaisseur variable au cours du mouvement, phénomène qui se trouve confirmé par les observations que nous avons pu observer. L'hypothèse d'approximation contenue dans cette définition concerne, non la vitesse, mais la dérivée de la vitesse dujdy. Cette hypothèse présente l'avantage de conserver un ròle symétrique au frottement du fluide sain sur la couche limite et au frottement de la paroi sur celle-ci, ce qui, ainsi que nous l'avons vu, est essentiel dans les mouvements étudiés.

\section{b) Solution de L'Équation De Navier-Stoles EN $u$ :}

En se bornant à la recherche d'une répartition des vitesses dans la couche limite $u / u_{\hat{\delta}}$ (1), satisfaisant à l'équation simplifiée de Navier-Stokes en $u$, qui s'écrit :

$$
\partial u^{*} / \partial t=(1 / y) \quad\left(\partial 2 u^{*} / \partial y^{2}\right)
$$

(1) Nous appelons $u_{\delta}$ la valeur de la vitesse du fluide sain a la frontière de la coucho limite en négligeant l'existence du cosinus; nous avons donc : vitesse à la frontière de la couche limite $=u_{\delta} \cdot \cos (b t-a x)$. 


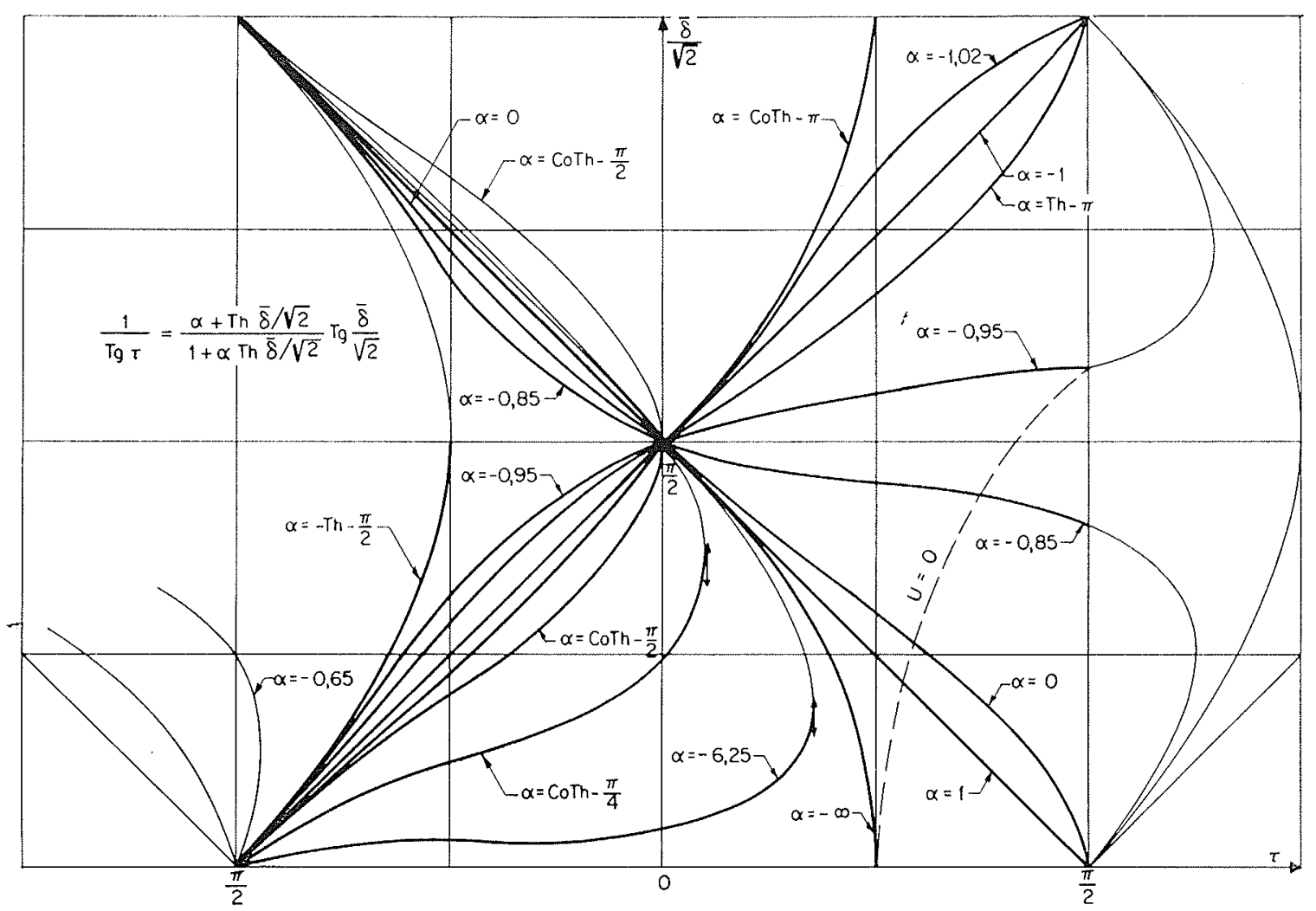

Fia. 1

en appelant $u^{*}$ la vitesse complémentaire de la vitesse du fluide sain, telle que $u_{c l}$ (vilesse de la couche limite $)=u^{*}+u_{f}$ (vitesse du fluide sain).

En se bornant aux termes du premier ordre en $y$, la solution de cette équation est (2) :

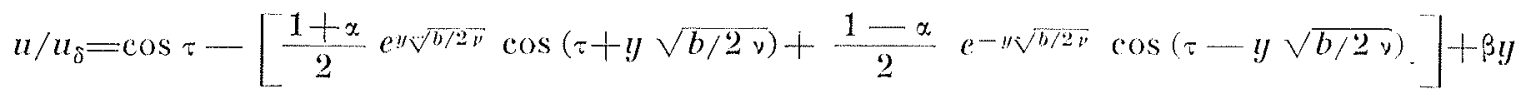
s'écrit :

L'équation déterminant la frontière de la couche limite à partir de l'équation ci-dessus

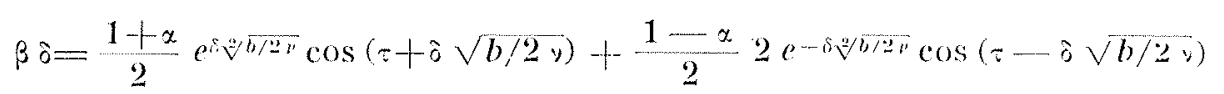

Nous avons étudié en détails cette équation dans le cas de $\beta=0$.

Les différentes courbes obtenues représentant l'épaisseur de la couche limite en fonction de $\tau$ permettent de rendre compte des différents phénomènes observés expérimentalement (fig. 1). L'existence du courant moyen d'entrainement correspondant à $\beta$ positif a pour résultats de diminuer l'épaisseur de la couche limite au cours du mouvement aller et de l'augmenter au cours du mouvement retour, ce qui est conforme à nos expériences. Enfin, à partir de cette détermination théorique, nous avons défini une épaisseur moyenne théorique de la couche limite laminaire correspondant aux points obtenus pour $\tau=k \pi, \delta_{t h} \sqrt{b / 2} \vee=\pi / 2$ et qui correspond à $: \delta_{t h}=\sqrt{v T / 2} \sqrt{\pi}$, c'est à dire à une valeur de $k$ dans la formule $\delta=k \sqrt{v t}$ égale à 1,80 .

\section{c) Etude phrsigue de la covche limite:}

Si l'étude mathématique de la couche limite est intéressante en ce sens qu'elle permet, d'une part, de vérifier un certain nombre de résultats d'observation, et, d'autre part, de justifier les hypothèses de définition, il est instructif de dé- crire le mouvement dans la couche limite à partir des hypothèses physiques qui ont été mises en évidence dans l'étude de la couche limite pour des écoulements relativement simples (écoulement permanent).

(2) Nous posons $b t-a x=\tau$. 
D'après les résultats classiques des couches limites dans le cas de la plaque plane, l'épaisseur de la couche limite, dans le cas d'un mouvement permanent, s'écrit [3]:

$$
\delta=4,92 \sqrt{v x / u_{0}}=4,92 \sqrt{v t}
$$

que nous écrirons :

$$
\delta=k \sqrt{y t}
$$

Nous nous trouvons, en fait, en présence de mouvements non permanents et il faut tenir compte de la variation de vitesse. Or, il est connu que l'accroissement des vitesses, dans le sens de l'écoulement, constitue un facteur de stabilité et que la décroissance des vitesses, dans le sens de l'écoulement, constitue un facteur d'instabilité. De même, un gradient de pression positif en allant de l'amont à l'aval de l'écoulement est un facteur d'instabilité, un gradient de pression négatif est un facteur de stabilité.

Ces influences entraînent un accroissement de l'épaisseur de la couche limite lors du mouvement retour et une diminution de celle-ci lors du mouvement aller par rapport à la loi parabolique.

Mais, de plus, la particularité des mouvements de houle réside dans la périodicité du mouvement en fonction de $(b t-a x)$; il en résulte une action du gradient de la vitesse verticale qui correspond à la fin du mouvement aller, à une succion de la couche limite (fig. 2) et, à la fin
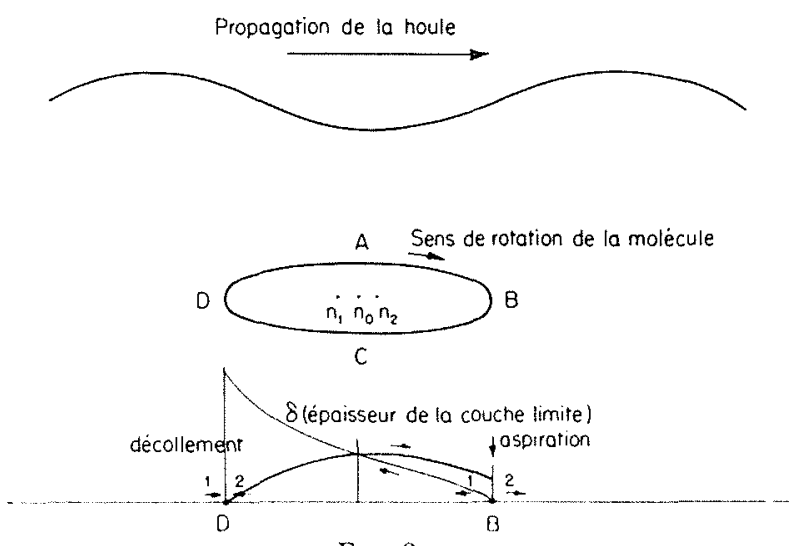

Fia. 2

du mouvement retour, à une injection de fluide sous la couche limite dans le cas du mouvement aller.

L'ensemble des facteurs ainsi envisagés permet donc de prévoir les phénomènes suivants :

- Un décollement de la couche limite est probable au voisinage des points $D$ ou $B$ (vitesse nulle parallèlement à l'écoulement);

- Le décollement en D s'obtiendra très facilement (injection dans la couche limite) alors que le décollement en $B$ ne s'obtiendra que dans des cas particuliers (succion de la couche limite);

- Le passage de la couche limite laminaire à la couche limite turbulente s'effectuera de préférence aux environs des points $A$ et $C$ (maximum de vitesse);

- Au contact de la paroi, la couche limite est douée d'un mouvement horizontal moyen important dans le sens de la propagation de la houle. En effet, d'une part, la couche limite est plus épaisse de $B$ en $\mathrm{D}$ que de $\mathrm{D}$ en $\mathrm{B}$, el, par conséquent, une part plus importante du fluide est ralentie par la présence de la paroi pour le mouvement de retour; d'autre part, le profil des vitesses correspondant a des valeurs de $u / u_{\delta}$ sont dans l'ensemble inférieures pour le parcours BD que pour le parcours $\mathrm{DB}$;

- Lorsqu'il y a décollement en D, l'effet d'aspiration vers le haut provenant de la présence de vitesses verticales ascendantes, ou l'effet d'injection provenant du facteur $d u / d x$ (les deux effets n'étant que des explications différentes du même phénomène) Lransforme, dans les expériences de colorimetrie, la couche limite laminaire en une corne qui vient mourir dans le fluide sain et peut introduire une turbulence partielle dans celui-ci (gradient de vitesse important);

- Ce phénomène et la rapidité de croissance de $\delta$ pour $\tau=\pi / 2+k \pi+\varepsilon$, justifient l'hypothese faite au début de ce raisonnement de la naissance d'une nouvelle couche limite à chaque renverse de sens du courant;

- Si l'on observe une couche limite en un point fixe (coordonnées d'Euler) et non en suivant une particule donnée, on observera une pulsation de la couche limite; on aura l'impression que « la couche limite respire».

\section{d) E'TUdE DU DÉCOLLEMENT :}

La méthode utilisée par Karl Pohlhausen pour létude du décollement des couches limites dans le cas de variation de vilesse le long de la couche limite [3] aurait pu etre appliquee avec le mème degré d'approximation que dans le cas de la plaque plane au cas des mouvements cylindriques qui nous intéressent. Les calculs de cet auteur l'amènent à introduire un coefficient de forme de l'écoulement $\Lambda=\left(\hat{\delta}^{2} / y\right)(\partial u / \partial x)$ que nous écrirons sous la forme :

$$
\Lambda=\left(\delta^{2} / v\right)(1 / u)(d u / d t)
$$

Les calculs de Pohlhausen ont montré que le décollement se produisait lorsque $\Lambda=-12$. Dans le cas du mouvement qui nous intéresse, 
$\Lambda$ a pour expression - $\left(\delta^{2} / y\right) b \operatorname{tg}(b t-a x)$. Le fait que $\operatorname{tg}(b t-a x)$ tende à devenir $\infty$ pour $b t-a x \rightarrow \pi / 2$ montre que la décélération en fin de mouvement - mouvement aller et retour correspond à une instabilité du mouvement $\Lambda=-\infty$. Nous pouvons toutefois admettre que le décollement ne sera sensible que s'il se produit pour des vitesses à la lrontière relativement importantes et en particulier nous pourrons admettre que dans le cas des houles le critère de Pohlhausen doit s'appliquer pour une valeur de $\operatorname{tg}(b t-a x)=1$. Dans ce cas, il n'y aura décollement à la fin du mouvement aller ou retour que si $\left(\delta^{2} / y\right) b \geqslant 12$. Les calculs montrent que ceci n'est possible que si $\hat{o} \geqslant \hat{o}_{\mathrm{th}} \times 1,6$, ce qui est vérifié dans le cas du mouvement retour, mais non dans le cas du mouvement aller.

\section{Conclusion}

L'ensemble de l'expérimentation, de l'étude mathématique et de l'étude physique du mouvement constitue un faisceau de faits qui justifie les considérations que nous avons exposées sur l'existence de la couche limite des mouvements progressifs périodicues el montre l'importance de ce problème dans l'étude de la houle.

\section{III. - DÉVELOPPEMENT ET STABILITÉ DES PHÉNOMENES DE COUCHE LIMITE}

Des études expérimentales que nous avons effectuées concernant le développement des phénomènes de couche limite, il a semblé intéressant d'essayer de déterminer les critères permettant de définir les différents phénomènes et leurs zones de stabilité.

Nous exposerons maintenant rapidement les résultats auxquels nous avons abouti.

\section{a) Couche limite totalement turbulente :}

A la suite des expériences effectuées au laboratoire de Berkeley, M. Huon $\mathrm{Li}$ avait proposé [4], pour caractériser la stabilité de la couche limite des mouvements périodiques, un critère basé sur l'observation d'oscillations alternées et périodiques du fond d'un réservoir. Nos expériences nous ont amenés à conclure que les critères proposés par M. Huon Li correspondaient à un domaine trop important de stabilité en ce qui concerne les mouvements de houle. Nos expériences nous ont amenés à caractériser la stabilité de la couche limite des mouvements de houle en fonction, d'une part, d'un paramètre sans dimension de l'écoulement:

$$
\mathrm{X}_{1}=\frac{b^{1 / 2}}{y^{1 / 2}} \frac{2 h}{\operatorname{ch} a \mathrm{H}}
$$

et, d'autre part, un paramètre sans dimension de la rugosité :

$$
\mathrm{R}_{\epsilon}=\frac{b^{1 / 2}}{\nu^{1 / 2}} \varepsilon
$$

D’après nos expériences, la couche limite totalement turbulente correspondrait au domaine défini par la relation :

$$
\mathrm{R}_{\epsilon} \mathrm{X}_{1}^{2,5} \geqslant \mathrm{C}^{\mathrm{te}}
$$

Il importe de remarquer que le paramètre sans dimension que nous utilisons pour caractériser l'écoulement diffère, par rapport à celui utilisé par M. Huon Li, par le remplacement de ch $a \mathbf{H}$ par sh $a \mathrm{H}$, au dénominateur.

Pour les houles usuelles en cau profonde, ces deux paramètres sont relativement égaux mais diffèrent sensiblement en eau moyennement profonde. Mais, en ce qui concerne l'extrapolation des critères de turbulence ci-dessus énoncés, au cas de la marée, le critère que nous avons adopté conduit à admettre que la couche limite de la marée est toujours turbulente.

\section{b) CoUche limite laminarRe, MOUVEMENTS PAR- TIELLEMENT TURBULENTS :}

Ainsi que nous l'avons expliqué ci-dessus, s'il est aisé de distinguer le domaine des mouvements turbulents du domaine des mouvements laminaires et des mouvements partiellement turbulents, il est, par contre, difficile de délimiter par un seul parametre sans dimension les domaines de ces deux mouvements.

Nous avons, en fait, été amenés à introduire un nouveau parametre $\mathrm{X}_{3}=(1 / v)(a / \mathrm{H})(d u / d y)$ qui caractérise l'influence du mouvement du fluide sur la couche limite. Nous verrons que ce même paramètre a permis de caractériser l'évolution de la turbulence dans le fluide sain dans le cas de la couche limite totalement turbulente. La probabilité d'observations du mouvement partiellement turbulent dépendrait, en effet, de l'influence relative de deux paramètres $\left(X_{1}\right.$ et $\mathrm{X}_{3}$ ) ainsi que des causes extérieures d'instabilité (rugosité, turbulence, etc.), le paramètre $\mathrm{X}_{1}$ caractériserait l'instabilité due aux forces de viscosité dans la couche limite; le paramètre $\mathrm{X}_{\text {: }}$ caractérise la stabilité due aux conditions im- 
posées par le mouvement du fluide sain, étant entendu que les fortes valeurs de $\mathrm{X}_{3}$ correspondent à une stabilité du mouvement et les faibles valeurs à une instabilité, tandis que c'est l'inverse qui se produit en ce qui concerne l'influence de $\mathrm{X}_{1}$.

\section{c) VITESSE de DÉPLACEMENT MOYEN DE LA COUCHE LIMITE :}

Ainsi que nous l'avons exposé, une des caractéristiques physiques essentielles de la couche limite dans les mouvements progressifs périodiques, réside dans l'existence d'une vitesse moyenne de déplacement de la couche limite, dans le sens de propagation de la houle.

L'existence de cette visite de la couche limite constitue en f'ait une loi générale du mouvement eylindrique progressif dans le cas de la couche timite laminare ou partiellement turbulente. D'après les résultats de nos expériences, nous avons trouvé qu'il était possible de définir la vitesse moyenne de déplacement de la couche limite près du fond, en fonction de la vitesse maximum théorique calculée des particules du fluide sain près de la paroi, dans le cas où le mouvement était laminaire ou partiellement turbulent.

Dans la limite de nos expériences, la formule:

$$
u_{c l}=k \frac{\left(2 u_{f}\right)^{1,6 ;}}{\mathrm{H}^{0,6}}
$$

dans laquelle :

$$
\begin{aligned}
& u_{a !} \text { : vitesse moyenne de la couche limite; } \\
& u_{f} \text { : vitesse calculée près du fond; } \\
& \mathrm{H} \text { : épaisseur de la lame d'eau; }
\end{aligned}
$$

permet de traduire d'une facon suffisamment approchée les résultats pour des rugosités de fond $\mathrm{s}$ pourant atteindre $0,5 \mathrm{~mm}$. Cette formule, essentiellement valable en fond horizontal, a été trouvée pouvoir traduire la vitesse de déplacement dans la couche limite laminaire pour des fonds de pente 1,2 et $5 \%$ tant que la rugosité s est $\leqslant 0,2 \mathrm{~mm}$. Toutefois, la dispersion des points d'expérience est dans ce cas plus accentuée.

\section{d) Vitesse MOYENNE D'ENTRAinement du FuUdDE SAIN AU-DESSUS DE LA COUCHE LIMITE:}

L'existence d'une vitesse moyenne dans la couche limite $\left(u_{c l}\right)$ se traduit dans le fluide sain par une distribution de rotationnels décroissant jusqu'en surface et corrélativement, par l'existence d'une vitesse d'entraînement moyen du fluide sain variable suivant la distance du point considéré par rapport au fond.
Pour tenir compte, dans les équations, de cette répartition de vitesse, il importe ae connaitre la valeur de cette vitesse pris du fond, cest-ádire la vitesse d'entraìnement moyen du liuide sain à la frontière de la couche limite $\left(u_{i}\right)$. Nous avons cherché à relier les vitesses observées à la frontiere de la couche limite à la vitesse moyenne de déplacement de la couche limite qui, ainsi que nous l'avons vu ci-dessus, peut ètre reliée aux caractéristiques propres du mouvement. Dans le domaine de nos expériences, la relation suivante:

$$
u_{k}=\frac{1}{1,6} u_{c l}
$$

permet de rendre compte de façon satisfaisante de la valeur de la vilesse moyenne d'entrainement du fluide sain à la frontière de la couche limite. Ces résullats permettent, en particulier, de définir lat répartition thérique des courants d'entrainement - connaissant ainsi la vitesse d'entrainement $\left(n_{e}\right)$ à la frontière de la couche limite -... si l'on connaît en plus la vitesse d'entrainement en surface.

Rappelons que, dans le cas des mouvements turbulents, les vitesses d'entrainement de la couche limite sont aléatoires et dépendent essentiellement de phénomènes autres que des phénomènes de frottement sur le fond.

\section{e) Epaisseur de la covche limite:}

Au cours des différents essais que nous avons effectués, nous avons mesuré l'épaisseur apparente de la couche limite, telle que nous l'avons précisée ci-dessus dans les différents cas où celleci était visible.

Nous avons comparé chacune de ces valeurs à la caractéristique de la couche limite laminaire telle qu'elle résulte des calculs exposés ci-dessus.

Cette épaisseur est donnée par l'expression :

$$
\delta_{t+1}=\pi \sqrt{ } \cdot 2 b
$$

\section{Conche limite laminaire:}

Dans le domaine de nos expériences, on peut affirmer que l'épaisseur de la couche linite laminaire observée est comprise entre 0 el 2 fois la valeur théorique caractéristiqque de la conche limite.

Il existe, pour de faibles valeur's de l'épaisseur théorique caractéristique, quelques points relatifs à une couche limite laminaire, correspondant à une épaisseur observée, supérieure à deux fois $\hat{o}_{\text {th }}$; mais il importe de rappeler que l'épaisseur de la source colorante peut introduire des erreurs de mesures, en particulier dans les cas où les vitesses de mouvement étant très faibles, la présence d'une source colorante de di- 
mension supérieure à celle de la couche limite, ne provoque pas immédiatement de turbulence partielle.

Etant donné, d'autre part, que l'épaisseur mesurée du front coloré dépendait également de la finesse du grain colorant, les valeurs de $\delta$ inférieures à l'épaisseur caractéristique théorique peuvent être considérées comme correspondant à l'observation d'une partie seulement d'une couche limite d'épaisseur sensiblement égale à l'épaisseur caractéristique.

D'apres ces remarques, on peut affirmer que les mesures effectuces justifient le calcul théorique exposé au paragraphe II et permettent d'affirmer que la zone influencée par la couche linite des mouvements laminaires, dans le cas des mouvements progressifs périodiques cylindriques, est comprise entre 1 et 2 fois la valeur caractéristique de la couche limite.

\section{Couche limite particllement turbulente:}

On peut admettre, en moyenne, que la zone intéressée par la turbulence diffuse à partir de la couche limite partiellement turbulente est comprise entre 2 et 8 fois l'épaisseur caractéristique théorique.

\section{Conche limite turbulente:}

Les mesures ont permis de mettre en évidence une croissance très rapide de l'épaisseur en fonction de l'épaisseur caractéristique théorique dans le cas de la couche limite turbulente.

Pour les valeurs de la rugosité étudiée $\varepsilon=3 \mathrm{~mm}$, on peut admettre que l'épaisseur de la couche limite turbulente est comprise en moyenne entre 15 fois et 60 fois l'épaisseur caractéristique théorique.

\section{Décollement de la couche limite:}

Nous avons également étudié la hauteur des décollements observés au cours de nos expériences en fonction de l'épaisseur effective de la couche limite. A l'exception de quelques valeurs correspondant à une couche limite d'épaisseur extrèmement faible rentrant dans le cadre du film laminaire partiel, on peut admettre que la hauteur maximum des décollements observés est comprise entre 1 à 3 fois la valeur de la couche limite. En fait, dès que la couche limite devient relativement épaisse, la hauteur des cornes par rapport a la couche limite se limite entre 1 fois et 1,5 fois l'épaisseur de celle-ci.

On peut donc admettre que la présence du décollement introduit une perturbation dans l'écoulement au-dessus de la couche limite pouvant atteindre de 1 à 3 fois l'épaisseur de celleci.

\section{f) EMISSION DE TURBULENCE daNS Le Fluide SAIN :}

Dans le cas de mouvements totalement turbulents, la couche limite se définit comme la zone dans laquelle l'action de la viscosité est telle que le gradient de vitesse entraine la transformation d'une partie de l'énergie du mouvement en énergie turbulente. Toutefois, les mouvements des houles ne correspondant pas à un déplacement moyen important des molécules, la turbulence dans la couche limite contamine l'écoulement du fluide sain, qui devient lui-même turbulent.

Mais, dans cette zone, la viscosité du fluide iniervient comme un facteur d'ćvolution de la turbulence et non comme un facteur générateur de turbulence. On observe, alors, une évolution vers une turbulence de plus en plus fine.

Nous avons observé l'émission de l'énergie turbulente et son évolution dans le fluide sain en étudiant la progression du nuage coloré audessus de la source colorante. Nous avons tracé la courbe d'évolution, en fonction du temps, de la hauteur atteinte par le front coloré, qui illustre la loi de variation du gradient de turbulence dans le fluide sain.

Pour pouvoir classer les différents phénomènes nous avons dû admettre que le facteur $\mathrm{X}_{1}$ pouvait caractériser l'importance de l'émission de turbulence, et que le paramètre $\mathrm{X}_{3}$ caractérisait au contraire l'influence évolutive du mouvement du fluide sain sur l'évolution de la turbulence.

\section{g) Influence de la rugosité :}

Les rugosités faibles inférieures ou égales à $0,1 \mathrm{~mm}$ sont absolument comparables en ce qui concerne l'épaisseur de la couche limite envisasée au cours des expériences. Par contre, les rugosités de 0,2 et de $0,5 \mathrm{~mm}$ correspondent, en général, à des couches limites légèrement plus importantes. Celles-ci sont d'ailleurs en général des couches limites partiellement turbulentes. Il semble donc que la valeur de la rugosité a une influence certaine sur la formation des mouvements à couche limite particllement turbulente.

On peut admettre, à la suite de nos résultats d'expérience, que la rugosité a une influence sensible dès que celle-ci est supérieure au quart de l'épaisseur théorique de la couche limite laminaire et qu'elle joue un ròle important dans la probabilité d'émission de turbulence diffuse.

En ce qui concerne les mouvements totalement turbulents, nous n'avons jamais observó de sous-film laminaire pour la rugosité $\varepsilon=3 \mathrm{~mm}$. Les sous-films laminaires que nous avons observés, en général, correspondent à des épaisseurs inférieures à l'épaisseur caractéristique théorique. On peut admettre comme ordre de grandeur général que le sous-film laminaire de la couche 
limite turbulente est le quart ou le cinquième de l'épaisseur caractéristique théorique.

Dans ces conditions, on peut admettre qu'en ce qui concerne les couches limites turbulentes, il existe un sous-film laminaire d'épaisseur $\delta b=0,2 \delta_{\text {tll }}$, tant que l'écoulement n'est pas hydrauliquement rugueux. La limite de l'écoulement hydrauliquement lisse correspondant à $\varepsilon / \delta b<1 / 3$, le fond sera considéré comme hydrauliquement rugueux et influencera l'épaisseur de la couche limite turbulente et la stabilité du sous-film laminaire lorsque :

$$
1 / 3>\varepsilon / \delta b>8
$$

\section{h) INFLUENCE DE LA PENTE DU FOND:}

L'étude de couches limites des houles sur un - fond non horizontal permet d'énoncer les conclusions suivantes:

a) L'existence d'une pente faible (1\%) semble constituer un facteur de stabilité de l'écoulement près du fond. Cette constatation peut s'expliquer par le fait que l'énergie dissipée par frottement équilibre sensible- ment l'augmentation d'énergie des zones profondes du mouvement provoqué par l'action de convergent du fond, vis-à-vis de la propagation de l'énergie;

b) L'existence d'une pente appréciable $(\geqslant 2 \%)$ semble constituer un facteur d'instabilité de l'écoulement. La valeur caractéristique du paramètre $X_{1}$ pour les couches limites totalement turbulentes, qui est d'environ 120 pour un fond horizontal et pour une r'ugosité de $\varepsilon=0,2 \mathrm{~mm}$, devient de l'ordre de 50 pour des pentes de $2 \%$ ou $5 \%$, pour la même rugosité;

c) Pour des valeurs de $\mathrm{X}_{\Perp}$ inférieures à la valeur caractéristique, une pente $\geqslant 2 \%$ semble constituer un facteur défavorable à l'émission de turbulence partielle;

d) Toutefois, à proximité du raccordement avec le fond horizontal, l'influence du paramètre que constitue la pente est beaucoup moins sensible. II semble que la courbure positive du fond constitue un élément de stabilité (résultat comparable à celui de la couche limite de la plaque plane).

\section{IV. - APPliCATIONS DES PHENOMENES DE COUCHE LIMITE AUX MOUVEMENTS DES MATÉRIAUX SOUS L'ACTION DE LA HOULE}

Certains phénomènes concernant les mouvements de matériaux sous l'action de la houle s'expliquent par extension des résultats propres à la couche limite que nous avons précédemment énoncés et constituent une application pratique de ces études théoriques.

Nous avons distingué dans nos études le cas des matériaux pulvérulents du type sable et le cas des matériaux du type vase.

a) Mouvements des matériadx de tyre sable:

Les mouvements de matériaux du type sable sous l'action de la houle peuvent revêtir trois aspects différents :

- Mouvements en surface des grains isolés;

- Formation de rides et mouvements des rides;

- Mouvements en masse du matériau par mise en saltation sous l'effet de la houle.

Parmi ces phénomènes nous avons, en particulier, étudié :

- la naissance et le processus de formation des rides de sable;

- l'équilibre apparent, l'évolution et les limites de stabilité des rides de sable.

Les phénomènes de couche limite et, en particulier, de décollement de celle-ci, permettent de donner une explication de la naissance et du processus de formation des rides de sable sous l'action de la houle. L'existence des rides transforme les phénomènes de la couche limite qui étaient précédemment, comme le mouvement de la houle, un phénomène périodique dans le temps et dans l'espace, en un phénomène stationnaire dans l'espace, périodique uniquement dans le temps. Ce processus détermine l'équilibre apparent des rides et explique la stabilité du système houle-rides de sable (avec, dans certaines conditions, cheminement lent des rides de sable).

Toutefois, pour des amplitudes de houle suffisamment importantes, cette stabilité est détruite par la mise en saltation du matériau sur une épaisseur notable, et on observe alors un transport considérable du matériau dans le sens de propagation de la houle, suivant un processus assimilable à celui du débit moyen de la couche limite des houles. Les résultats expérimentaux peuvent être donnés en fonction de paramètres sans dimension formés à partir des caractéristiques des houles. On peut expliquer aussi certains phénomènes, observés sur les plages de sable, mais dont la cause profonde du point de vue hydraulique n'a jamais été analysée, à notre connaissance. 


\section{b) Mouvements des Matériaux du TyPe vase :}

Dans l'étude que nous avons effectuée, nous sommes toujours restés dans le cas d'une amplitude de houle insuffisante pour provoquer la mise en suspension du matériau vaseux déposé sur le fond. Toutefois, l'amplitude du mouvement était suffisante pour exercer une influence non négligeable sur le matériau vaseux. On observe alors, dans la propagation du mouvement, une modification des propriétés de la couche limite, par suite de la répartition des efforts de viscosité entre la couche limite proprement dite du fluide et le milieu vaseux.

La propagation du mouvement dans le milieu vaseux varie en fonction de la viscosité relative et de la rigidité initiale du matériau, mais, d'autre part, l'influence des propriétés thixotropiques caractéristiques de la vase provoque une diminution progressive de la viscosité relative et entraine une augmentation du mouvement en profondeur au cours du temps, jusqu'à l'obtention d'un certain équilibre.

Un phénomène particulier caractéristique de la propagation d'un mouvement à caractère progressif et périodique dans un milieu de viscosité importante tel que la vase, consiste dans le fait que l'on observe, comme dans le cas de la couche limite, un débit non négligeable dans la direction de propagation de la houle. Il s'ensuit une remontée de matériau vers la partie aval du canal d'expérimentation entrainant, soit la formation d'une plage de vase, soit la remise en suspension de la vase dans la zone de déferlement.

\section{CONCLUSION}

L'étude que nous avons entreprise avait pour but de réunir des résultats d'ensemble concernant l'importance des phénomènes de viscosité près du fond, en ce qui concerne la propagation de la houle, et son action sur les fonds.

Cette étude s'est limitée au cas des houles progressives cylindriques monochromatiques, qui constituent la représentation la plus simple des mouvements progressifs périodiques.

Les phénomènes marginaux, que nous avons étudiés, et qui concernent le développement des couches limites près du fond sous leurs différents aspects : laminaire, partiellement turbulente, ou totalement turbulente, ont permis de mettre en évidence certaines propriétés caractéristiques fondamentales de la propagation de ces mouvements.

L'étude de ces phénomènes présente un grand intérêt et apporte des éléments nouveaux, en ce qui concerne les mouvements de la houle et ses conséquences. Les progrès nouveaux concernant la connaissance de ces mouvements semblent devoir être recherchés essentiellement dans l'introduction des phénomènes marginaux, pour lesquels les équations du fluide sain, jusqu'ici développées, ne sont pas suffisantes, les termes de viscosité ne pouvant être négligés.
Plusieurs études partielles sur ces différents phénomènes que nous avons analysés au cours de notre étude, montrent l'intérêt qui s'attache à une meilleure connaissance de ceux-ci.

Le domaine d'application pratique de ces recherches, expérimentales ou théoriques, est extrêmement vaste, et comprend par exemple :

- Les problèmes de similitude et en particulier la reproduction correcte de la houle sur modèle réduit;

- L'amortissement des houles en nature et en laboratoire:

-.- L'action des houles sur les matériaux;

- L'équilibre des plages;

- La formation des polders, el la remontée des vases marines dans les estuaires. etc.

De cet ensemble de problèmes extrêmement vaste, nous avons cherché à établir une première théorie constituant un aperçu général, que les expériences futures, et, en particulier, les mesures en nature, doivent permettre de compléter, d'élargir et de vérifier. Des vérifications nombreuses des théories ci-dessus exposées, sont possibles dans le domaine du laboratoire, et il serait souhaitable qu'elles soient effectuées dans l'avenir.

\section{BIBLIOGRAPHIE}

(1) Biesel (F.). - Calcul de l'amortissement d'une houle dans un liquide visqueux de profondeur finie. (La Honille Blanche, 1956, $\mathrm{n}^{\circ}$ 5.)

(2) Breser (F.) et Canry (C.). - A propos de l'amortissement des houles dans le domaine de l'eau peu profonde. (La Houlle Blanche, 1956, $\mathrm{n}^{\prime \prime}$ 6.)

(3) Bron (E,). Introduction à l'étude de la couche limite. (Gauthiers-Villars, 1955.)
(4) Hoon Lr. - Stability of oscillatory laminar flow along a wall. Beach Erosion Board; Technical memorandum, n" 47 (1954).

(5) Lamb. - Hydrodynamics. New York, Dover, 1945.

(6) Lhermite (P.). - Etude de la couche limite dans le cas des mouvements progressifs périodiques. Comptes rendus des séances de l'Academie des Sciences, 1957 , tome 244 , p. 2352. 
(7) Longuet-Higins. - Transports de masse dans la houle. Phil. Trans. Roy. Soc. of London, Série A, $n^{\circ} 903$, v. 245.

(8) Mrowe (R.). - Mouvements ondulatoires de la mer en profondeur croissante ou décroissante. Anmales des Ponts et Chalussées, 1942.

(9) Miche (R.). - Propriétés des trains d'ondes océanicques et de laboratoire. (C.O.E.C., n" 135 . Imprimerie Nationale, 1954.)
(10) Mras (R.). - Amortissement des houles dans le domaine de l'eau peu profonde. (Lat Honille Blanche, $1956, \mathrm{n}^{\circ} 5$.)

(11) Townsend (A.A.). - The structure of turbulence shear flow, Cambridge Universitl Press, 1956.

(12) Valembors. - La considération sur la similitude dans les essais de houles sur modele. (IV Journées de l'Hydraulique, Paris, 1956.)

\title{
DIS CUS SION
}

\author{
prósident : M. Gunat
}

M. le President souligne l'etude du probleme abordé par M. LHen II'Te et l'importance de ses conséquences, en particulier celle qui a trait a mouvement des materiaux.

M. Valembors précise que la thérie de la couche limite simple, qui considere le mouvement du fluide sain comme horizontal et non elliptique, se trouve dejà dans Lamb et que Keulegan avait retrouvé la même formule pour la houle en étudiant le développement de la couche limite sur une certaine longueur qui correspondait à l'oscillation.

M. Lhernitre lemarque que Lamb avait traité le cas général de l'excitation d'une masse fluide sous l'effet d'une force périodique, mais il n'a jamais prétendu l'appliquer aux cas des houles, ce que M. Huon Li a fait.

Au sujet du courant général d'entrainement par la houle, M. Valeubors rappelle que, d'après les exposés de Mit Kravtchenio, Fontaner et Daubertr, le courant indiqué par la thérie est indéterminé. Dans ces conditions, la question qui se pose aux physiciens est de savoir si la nature, parmi toutes les solutions possibles, en choisit certaines ou non.

Pour cela, des études experimentales ont été effectuées en Angleterre par Russel et Osorio et présentées au dernier congrès du « Coastal Engineering » en Flovide. Les auteurs ont fait varier, dans une houle en canal, les conditions aux limites, et ils ont mesuré le courant d'entrainement. Ils ont observé qu'en canal, il n'y avait indétermination que pour les valeurs faibles du paramètre $2 \pi d / \mathrm{L}$ ( $d=$ profondeur d'eau, $L=$ longueur d'onde de la houle). Pour les valeurs intermédiaires, la distribution des vitesses observées concordait avec celle calculce par Longuet-Higgins, tandis que pour les grandes profondeurs, la distribution obtenue etait celle de Stokes. Il n'est pas absolument sûr que l'on puisse étendre ces résultats à la houle naturelle, leffet d'echelle pouvant ètre important sur les grandeurs annexes qui risquent d'avoir une grande importance sur lindétermination de la distribution des vitesses (la viscosite par exemple).

M. BIEsEl mppelle qu'en l'absence de houle, c'estaudire dans le cas d'un fluide parfait sur un fond horizontal, on trouve un rotationnel indetermine : toutes les tranches horizontales peuvent se déplacer les unes par rapport aux autres à des vitesses différentes. Ceci est la raison profonde de lindetermination que l'on trouve dans les solutions. Mais lorsqu'on introduira, soit la viscosité, soit la turbulence, ce qu'on ne sait pas faire entièrement thériquement, l'indétermination ne subsistera plus, et c'est ce qui explique que l'on retrouve les résultats de Longuet-Higgins, au moins dans un certain domaine, puisque précisément il a tenu compte de la viscosite.

M. Lacome attire l'attention sur l'intérêt exceptionnel du travail de M. LHEnMrTe au sujet de l'entrainement des matériaux sur les plages et de la formation des rides, probablement aussi de leur migration, en somme sur l'étude du régime des côtes.

M. Ferrandon remarque que la définition conventionnelle de la couche limite se rapporte a champ des vitesses. Il pense qu'il y aurait intérêt à la choisir à partir de considerations énergetiques: en appelant couche limite la tranche sus-jacente au fond, dans laquelle l'énergie dilapidée sous forme thermique, du fait de la viscosité, est une quote-part donnée $(9 / 10$ ou 99/100) de l'énergie dilapidée dans les mèmes conditions dans la hauteur totale de la conche.

Il signale qu'en ce qui concerne un phénomène voisin de celui étudié actuellement (courants marins de derive), l'élaboration d'un exereice scolaire (a l'Eeole Polytechnique) lui a montré que cette méthode réussit parfaitement et conduit a des interprétations sensibles des phénomènes en jeu.

M. LHenstrte indique qu'il n'a etudie qu'une solution approchée de la conche limite ef qu'une solution exacte impliquerait lintroduction de conditions aux limites, à la frontière de la couche limite, qui exigent d'introduire les développements en série du mouvement du fluide sain jusqu'au terme du second ordre au minimum. II $\mathrm{y}$ a là une difficulte très importante et on peut être tenté de résoudre le problème par la méthode énergétique : c'est ce qui conduit M. LHensmate à introduire une solution approchée comprenant deux parametres $\alpha$ et $\beta$, dont $\alpha$ représente l'importance de l'action du movvement du fluide sain sur la couche limite par rapport a celle du fond et $\beta$ correspond à un mouvement d'entrainement croissant depuis le fond jusqu'a la face superieure de la couche limite.

La solution définitive consiste à déterminer la relation existant entre $\alpha$ et $\beta$ à partir des hypothèses énergétiques, c'est-iz-dire en ecrivant que la fonction dissipative est minimum.

M. Valembors estime quil $y$ a tout de mème une difficulté à traiter l'écoulement dans son ensemble, et qu'il est plus simple, comme on le fait en général en mécanique des fluides, de traiter séparément l'écoulement général et la couche limite, comme M. Busser l'a d'ailleurs fait dans ses travaux sur ce sujet. Pour la mème raison, M. VALenisors ne croit pas que le rapport de l'énergie dissipée dans la couche limite à l'énergie dissipee dans l'ensemble soit très significatif : ce rapport doit varier en effet, dans d'assez larges limites, suivant la profondeur relative ef sans que la définition de la couche limite soit differente, puisque le profil des vitesses varie de façon importante avec la profondeur relative.

M. Fennaxdon declare qu'il ne comprend pas trés bien l'objection de M. Valemmors. Il considere qu'une notion rattachee à l'énergétique convient mieux qu'une notion de cinématique pour caractériser des phénomènes irréversibles, tels que la viscosité qui est à la base de l'idée de couche limite.

M. le Président remercie très vivement M. LHerMare. 